\title{
RECOVERIES OF BRONZED GRACKLES BANDED IN SASKATCHEWAN
}

by C. Stuart Houston, 863 University Drive, Saskatoon (from files of Canadian Wildlife Service)

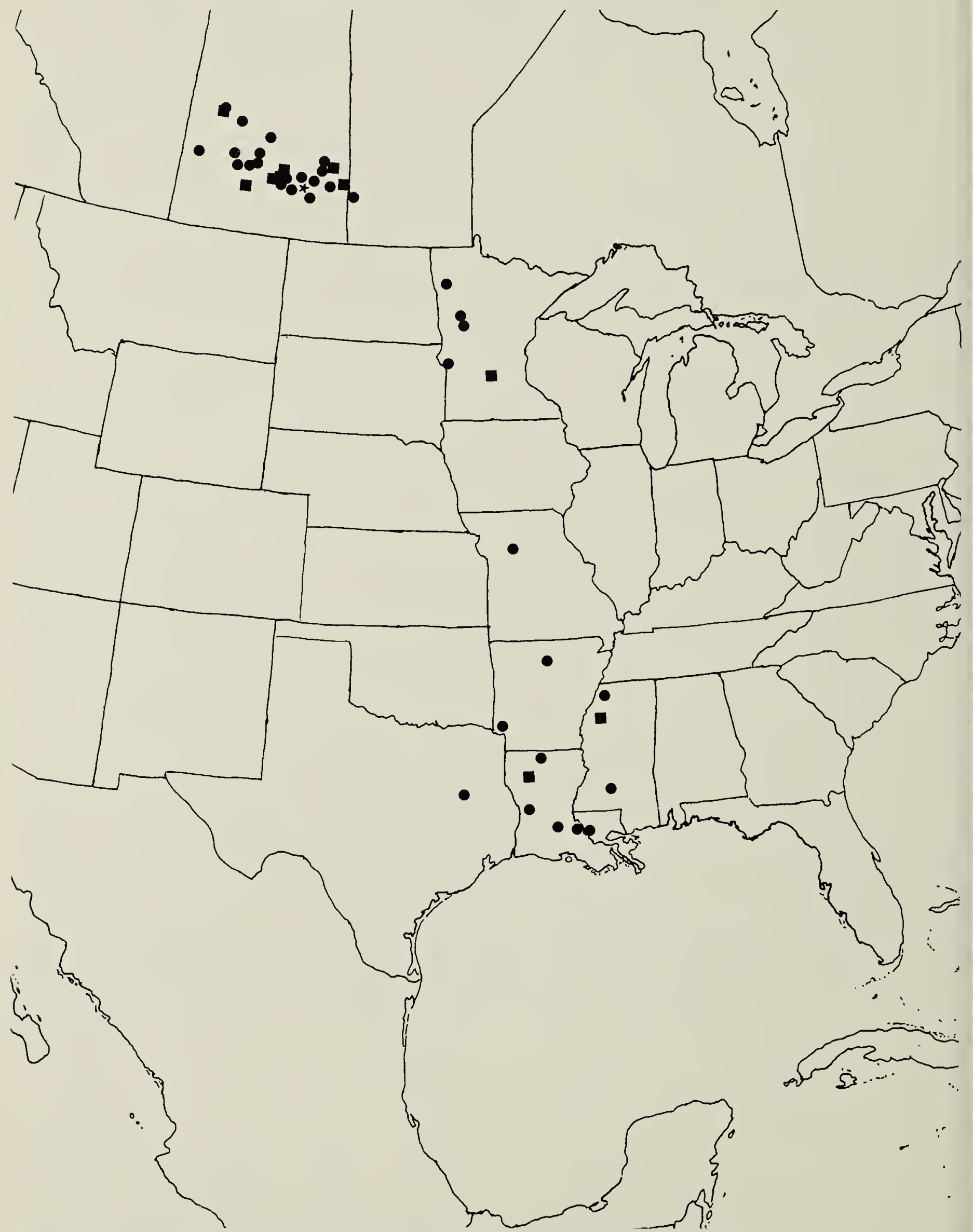

Recoveries of Bronzed Grackles banded in Saskatchewan (excluding those recovered at banding locality). Note: Squares represent direct recoveries (same year). Circles represent subsequent years. Star represents more than one recovery at a given location. 
From the recoveries to date, Saskatchewan grackles seem to have a remarkably narrow migration pathway, following the west side of the Mississippi River to winter in Texas, Louisiana and Arkansas. Of the 19 recoveries outside Saskatchewan, only three were "direct" (the same year), although another five occurred early in the next year before the grackles had returned north.

The writer's own recovery rate of $6.3 \%$ is remarkably high and comparable to the $5.3 \%, 6.3 \%$ and $8.8 \%$ obtained by Lloyd, Carter and Carter respectively and much higher than Lang's $2.8 \%$. This is surprising since band recovery rates tended to be significantly higher in the earlier years of banding than they are now. Perhaps this is compensated for by the early banders having included many nestlings which failed to reach maturity and by a higher recovery rate for grackles banded in cities than in rural areas. Note how many of the grackles have been shot, even in recent years.

In addition to the recoveries, there is a further category called "returns"; these are birds retrapped by the bander at the same locality in succeeding years. John R. Carter had three 1-year returns at Muscow; Bard had two 1-year returns at Regina; Houston at Yorkton had three at 1 year, three at 2 years and two at 4 years; at Saskatoon, four at 1 year, one at 2 years and one at 4 years.

Previous species (hawks and colonial birds) reported in this series needed no age designation since all were banded as flightless young. In the following table it is necessary to designate A for adult, I for immature and $U$ for unknown age. Within Saskatchewan, only recoveries outside the 7 by 11 mile local quadrant have been mapped and listed in detail; even then, a star had to be used to represent five circles at Qu'Appelle-Fort Qu'Appelle.

Banded by Reuben Lloyd, Davidson, Sask. (511-1055) (1.68 banded):

June 19/23. U. Retrapped May 24/24 (-1 yr.) Ortonville, Minn. (451-0962).

Aug. 1/23. U. In building Aug. 4/25 (2 yr.) Kenaston, Sask. (513-1061).

June 8/27. I. Drowned July 19/29 (2 yr.) Loreburn, Sask. (511-1063).

July 22/29. U. Drowned July 17/32 (-3 yr.) Bladworth, Sask. (512-1060).

Plus the following local recoveries: 1 shot, 1 killed by cat and 1 found dead the same year; 2 found dead the following year.

Banded by George H. Lang, Indian Head (503-1034) (461 banded):

June 13/24. U. Shot March 20/25 (-1 yr.) Lockesburg, Ark. (335-0940).

July 21/26. A. Band found Dec. 22/29 (3 yr.) Leesville, La. (310-0931).

June 26/27. I. Shot April/30 (-3 yr.) Sintaluta, Sask. (502-1032).

June 23/28. I. Shot May 20/33 (-5 yr.) Sintaluta, Sask. (502-1032).

June 7/32. I. Shot Oct. 23/32 (direct) Hutchison, Minn. (445-0942).

Plus the following local recoveries: 2 shot, 3 killed by cat and 4 found dead the same year; 1 band found over 3 years later.

Banded by R. H. Carter, Jr., Muscow, Sask. (504-1035) (80 banded):

July 12/24. A. Band found late June/27 (-3 yr.) Lemberg (504-1031).

Aug. 7/27. A. By dog late May/28 (-1 yr.) Lipton, Sask. (505-1035).

May 20/28. A. Shot Sept. 3/29 (1 yr.) Zehner, Sask. (503-1042).

May 27/28. A. Shot Jan. 17/31 (-3 yr.) Bernice, La. (324-0924).

July 6/30. I. Found dead April 17/33 (-3 yr.) Carrollton, Mo. (392-0933).

Banded by Philip Siemens, Hepburn, Sask. (523-1064):

May 1/28. A. Injured June 27/29 (1 yr.) North Battleford (524-1081).

May 10/29. A. Found dead Aug. 1/33 (4 yr.) Borden, Sask. (522-1071).

Banded by John R. Carter, Muscow, Sask. (504-1035) (91 banded) :

May 5/29. A. Shot Dec. 23/29 (direct) Hall Summit, La. (321-0931).

Apr. 28/29. A. Shot April 30/33 (4 yr.) Fit. Qu'Appelle (504-1034). 
May 5/29. A. By cat June 7/30 (1 yr.) Qu'Appelle (503-1035).

June 10/29. A. Found dead June 28/30 (1 yr.) Ft. Qu'Appelle, Sask.

Apr. 20/30. A. Shot July 4/31 (1 yr.) Qu'Appelle, Sask. (503-1035).

Apr. 27/30. A. Shot Dec. 1/32 (2 yr.) in Texas (314-0953).

May 14/30. A. Shot July 11/31 (1 yr.) Fort Qu'Appelle (504-1034).

Plus one local recovery: one shot a year later.

Banded by J. A. Briggs, probably NE of Regina, Sask.

June 6/30. A. Shot April 19/33 (-3 yr.) in Minnesota (463-0953).

Banded by Bill and Lindsay Wotherspoon, Hyas (515-1021) (7 banded) :

One local recovery: banded May 4/34; caught in muskrat trap May 10/35.

Banded by J. H. Goosen, Dalmeny, Sask. (522-1064):

One local recovery: banded May 11/35; shot June 11/36.

\section{Banded by Fred G. Bard}

Near Meota, Sask. (530-1082):

June 14/35. A. Shot Sept. 19/36 (1 yr.) in Minnesota (464-0954).

June 18/38. I. Shot June 25/38 (direct) N. Battleford (524-1081).

June 18/38. I. Sick May 21/39 (-1 yr.) Regina, Sask. (502-10-43).

Plus the local recoveries: 2 shot the same year and one shot 3 years later; band found 2 yrs. later.

Near Valeport, Sask. (504-1045):

June 9/37. A. Drowned late July/37 (direct) W. of Gibbs (505-1050).

Near Regina, Sask. (502-1043):

July 16/36. I. Shot early Oct./36 (direct) Strasbourg (510-1045).

June 5/37. A. Drowned Aug. 11/39 (2 yr.) Valeport, Sask. (504-1045).

June 12/38. I. Shot July 30/38 (direct) Vialeport, Sask. (504-1045).

June 16/38. A. Shot late July/40 (2 yr.) Valeport, Sask. (504-1045).

June 12/38. A. Found dead Feb. 3/40 (-2 yr.) in Louisiana (301-0910).

Banded by F. J. H. Fredeen, Macrorie, Sask. (511-1070):

Aug. 24/39. U. Shot Dec. 1/41 (2 yr.) in Arkansas (355-0922).

Aug. 26/39. U. Band found Jan. 31/40 (-1 yr.) in Mississippi (313-0900).

Aug. 26/39. U. Found dead Oct./40 (1 yr.) Surbiton, Sask. (512-1071).

Banded by C. Stuart Houston (460 banded)

At Yorkton, Sask. (511-1022):

July 15/46. I. Found dead Aug. 25/46 (direct) Ebenezer (512-1022).

Aug. 7/46. I. Found dead June 14/47 (-1 yr.) Lestock, Sask. (511-1035).

Aug. 8/47. I. Shot Sept. 14/47 (direct) Langenburg, Sask. (505-1014).

May 22/50. A. Shot before Dec. 29/50 (direct) Webb, Miss. (335-0902).

Sept. 16/51. I. Shot June 16/53 (-2 yr.) Springside, Sask. (512-1024).

July 4/52. I. Shot May 3/53 (-1 yr.) Birtle, Manitoba (502-1010).

July 15/52. I. Shot Feb. 17/55 (-3 yr.) Baton Rouge, La. (302-09:11).

July 26/52. U. Shot late Dec./54 (2 yr.) Euclid, Minn. (475-0963).

Aug. 20/54. U. Injured June 23/56 (-2 yr.) Bangor, Sask. (504-1022).

Plus the following local recoveries: 2 found dead the same year; found dead 1 , 2 and 4 years later ard band found 2 years later.

At Saskatoon, Sask. (520-1063):

May 2/61. A. By cat May 16/64 (3 yr.) Meacham, Sask. (520-1054).

May 14/61. A. Shot April 21/63 (-2 yr.) Kindersley, Sask. (512-1090).

June 3/62. A. Shot Jan. 24/63 (-1 yr.) Senatobia, Miss. (343-0900).

Sept. 13/63. U. Shot June 14/64 (-1 yr.) Asquith, Sask. (520-1071).

Sept. 25/63. I. Found dead Oct. 5/63 (direct) NE of Ernfold (503-1064).

Apr. 28/64. A. Shot Jan. 23/66 (-2 yr.) Ville Platte, La. (303-0921).

Plus the following local recoveries: 4 found dead the same year; found dead one year later; hit object one year later; shot 1 and 2 years later.

NOTE: 303-0921 means $30^{\circ} 30^{\prime}$ north and $92^{\circ} 10^{\prime}$ west. 